\title{
M31N 2008-05d: a M 31 disk nova with a dipping supersoft X-ray light curve ${ }^{\star}$
}

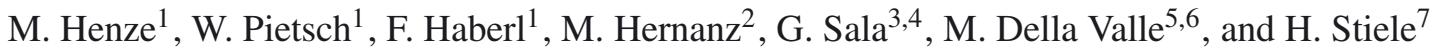 \\ 1 Max-Planck-Institut für extraterrestrische Physik, Postfach 1312, Giessenbachstr., 85741 Garching, Germany \\ e-mail: mhenze@mpe.mpg.de \\ 2 Institut de Ciències de l'Espai (CSIC-IEEC), Campus UAB, Fac. Ciències, 08193 Bellaterra, Spain \\ 3 Departament de Física i Enginyeria Nuclear, EUETIB, Universitat Politècnica de Catalunya, c/ Comte d’Urgell 187, \\ 08036 Barcelona, Spain \\ ${ }^{4}$ Institut d'Estudis Espacials de Catalunya, c/Gran Capità 2-4, Ed. Nexus-201, 08034, Barcelona, Spain \\ 5 INAF - Napoli, Osservatorio Astronomico di Capodimonte, Salita Moiariello 16, 80131 Napoli, Italy \\ ${ }^{6}$ International Centre for Relativistic Astrophysics, Piazzale della Repubblica 2, 65122 Pescara, Italy \\ 7 INAF - Osservatorio Astronomico di Brera, via E. Bianchi 46, 23807 Merate (LC), Italy \\ Received 26 April 2012 / Accepted 24 June 2012
}

\section{ABSTRACT}

\begin{abstract}
Context. Classical novae $(\mathrm{CNe})$ represent a major class of supersoft X-ray sources (SSSs) in the central region of our neighbouring galaxy M31. Significantly different SSS properties of CNe in the M31 bulge and disk were indicated by recent X-ray population studies, which however considered only a small number of disk novae.

Aims. We initiated a target of opportunity (ToO) program with XMM-Newton to observe the SSS phases of CNe in the disk of M 31 and improve the database for further population studies.

Methods. We analysed two XMM-Newton ToO observations triggered in Aug. 2011 and Jan. 2012, respectively, and extracted X-ray spectra and light curves.

Results. We report the discovery of an X-ray counterpart to the M 31 disk nova M31N 2008-05d. The X-ray spectrum of the object allows us to classify it as a SSS parametrised by a blackbody temperature of $32 \pm 6 \mathrm{eV}$. More than three years after the nova outburst, the X-ray light curve of the SSS exhibits irregular, broad dip features. These dips affect primarily the very soft part of the X-ray spectrum, which might indicate absorption effects.

Conclusions. Dipping SSS light curves are rarely observed in M 31 novae. As well as providing an unparalleled statistical sample, the M 31 population of novae with SSS counterparts produces frequent discoveries of unusual objects, thereby underlining the importance of regular monitoring.
\end{abstract}

Key words. galaxies: individual: M 31 - novae, cataclysmic variables - X-rays: binaries - stars: individual: M31N 2008-05d

\section{Introduction}

Classical novae $(\mathrm{CNe})$ originate from thermonuclear explosions on white dwarfs (WDs) in cataclysmic binary systems (see e.g. Bode \& Evans 2008). Accreted hydrogen-rich material accumulates onto the WD surface under degenerate conditions until hydrogen ignition starts a thermonuclear runaway. The resulting expansion of the hot envelope increases the optical brightness of the WD dramatically within from hours to days and leads to the ejection of mass at high velocities.

During the optical nova outburst, a fraction of the accreted material can remain on the WD surface under steady hydrogen burning (Starrfield et al. 1974). This powers a supersoft X-ray source (SSS) that becomes visible, once the opacity of the ejected matter reduces sufficiently (Starrfield 1989). These observational SSS turn-on timescales range from several weeks to even years (Pietsch et al. 2007; Schwarz et al. 2011). The duration of the SSS phase, of typically years (Krautter 2008), is limited by the amount of hydrogen fuel left on the WD and the SSS turn off marks the cessation of the hydrogen burning (Sala \& Hernanz 2005; Hachisu \& Kato 2006, 2010).

* Based on observations with XMM-Newton, an ESA Science Mission with instruments and contributions directly funded by ESA Member States and NASA.
Only X-ray observations are able to directly detect the hot WD photosphere and therefore provide important information on the physical parameters of the explosion, such as ejected and burned hydrogen mass (Sala \& Hernanz 2005; Hachisu \& Kato 2006). Estimates of the accreted and ejected masses might help us to determine the fraction of WDs in $\mathrm{CNe}$ that accumulate mass over time and ultimately become type Ia supernovae.

The existence of two distinct $\mathrm{CN}$ populations was first suggested by Duerbeck (1990) and Della Valle et al. (1992) based on data on Galactic novae. Fast novae (which have a time of decline by two magnitudes from maximum magnitude $t_{2} \leq 12 \mathrm{~d}$ ) are mainly associated with the disk of the Galaxy or are concentrated close to the Galactic plane, whereas slower novae are mostly present in the bulge region of the Galaxy or at greater distances from the Galactic plane (Della Valle \& Livio 1998).

Novae in the central region of our neighbouring galaxy M 31 (distance 780 kpc; Holland 1998; Stanek \& Garnavich 1998) have been found to constitute the major class of SSSs in this area (Pietsch et al. 2005). Owing to its proximity to the Galaxy as well as a moderate Galactic foreground absorption $\left(N_{\mathrm{H}} \sim 6.7 \times 10^{20} \mathrm{~cm}^{-2}\right.$, Stark et al. 1992), M 31 is a unique target for $\mathrm{CN}$ surveys in the optical and X-ray regime.

Recently, Henze et al. (2011, hereafter HPH2011) published a catalogue of 60 novae in M 31 with X-ray counterparts, 
Table 1. XMM-Newton EPIC observations of nova M31N 2008-05d.

\begin{tabular}{lccccccc}
\hline \hline ObsID & $\begin{array}{c}\text { Exp. time }^{a} \\
{[\mathrm{ks}]}\end{array}$ & $\begin{array}{c}\text { Date }^{b} \\
{[\mathrm{UT}]}\end{array}$ & $\begin{array}{c}\mathrm{MJD}^{b} \\
{[\mathrm{~d}]}\end{array}$ & $\begin{array}{c}\Delta t^{c} \\
{[\mathrm{~d}]}\end{array}$ & $\begin{array}{c}\text { Count Rate } \\
{\left[\mathrm{ct} \mathrm{s}^{-1}\right]}\end{array}$ & $\begin{array}{c}\mathrm{L}_{0.2-1.0^{d}} \\
{\left[\mathrm{erg} \mathrm{s}^{-1}\right]}\end{array}$ & $\begin{array}{c}L_{50}{ }^{e} \\
{\left[\mathrm{erg} \mathrm{s}^{-1}\right]}\end{array}$ \\
\hline 0560180101 & $20.0(17.4)$ & $2008-07-18.26$ & 54665.26 & 51 & $<2.8 \times 10^{-3}$ & $<0.9 \times 10^{37}$ & $<1.3 \times 10^{36}$ \\
0655620301 & $20.0(6.6)$ & $2011-08-01.26$ & 55774.26 & 1160 & $(4.0 \pm 0.4) \times 10^{-2}$ & $(1.7 \pm 0.2) \times 10^{38}$ & $(2.0 \pm 0.2) \times 10^{37}$ \\
0655620401 & $30.2(10.7)$ & $2012-01-21.76$ & 55947.76 & 1334 & $(4.7 \pm 0.2) \times 10^{-2}$ & $(2.0 \pm 0.1) \times 10^{38}$ & $(2.2 \pm 0.1) \times 10^{37}$ \\
\hline
\end{tabular}

Notes. ${ }^{(a)}$ EPIC pn total exposure time, in brackets we give the "good" time, corrected for dead time and high background, which was used to estimate count rates and extract the image in Fig. 1; ${ }^{(b)}$ start date of the observation; ${ }^{(c)}$ time in days after the outburst of nova M31N 2008-05d in the optical on 2008-05-28.04 (MJD = 54614.04; see Ovcharov et al. 2008); ${ }^{(d)}$ X-ray luminosities (unabsorbed, blackbody fit, 0.2-1.0 keV) and upper limits were estimated according to Sect. 3 (luminosity errors are $1 \sigma$, upper limits are $3 \sigma$ ); ${ }^{(e)}$ unabsorbed equivalent luminosity computed from $0.2-10.0 \mathrm{keV}$ count rates assuming a $50 \mathrm{eV}$ blackbody spectrum with Galactic foreground absorption of $6.7 \times 10^{20} \mathrm{~cm}^{-2}$.

a number that is significantly larger than for any other Galaxy, including the Milky Way. Using their catalogue, HPH2011 were able to present the first evidence of differing X-ray properties of M31 novae in the bulge and disk. However, small sample sizes, in particular for the relatively neglected disk novae, made those discoveries only weakly significant.

The XMM-Newton observations this work is based on were obtained using a target of opportunity $(\mathrm{ToO})$ programme aimed specifically at disk novae in M31. Thereby, we intended to increase the knowledge about this population as well as the database for further statistical studies.

Nova M31N 2008-05d was discovered in the optical by Ovcharov et al. (2008) on 2008-05-28.04 UT and confirmed as a nova by Henze et al. (2008b) using $\mathrm{H} \alpha$ observations. Furthermore, Henze et al. (2008a) reported optical observations indicating that the object is a slow nova that still showed increasing brightness on 2008-06-09.48 UT. In this work, we report on X-ray observations of M31N 2008-05d with XMM-Newton.

\section{Observations and data analysis}

A south-east region of the M 31 disk was observed with two XMM-Newton (Jansen et al. 2001) ToO pointings in August 2011 and January 2012 (see Table 1). Having been scheduled at the end of their revolutions, those observations suffered from high background radiation.

We carried out the screening and processing of data from the XMM-Newton European Photon Imaging Camera (EPIC; see Strüder et al. 2001; Turner et al. 2001) according to the methods described in Henze et al. (2010) using XMMSAS (XMM-Newton Science Analysis System; Gabriel et al. 2004) ${ }^{1}$ v11.0 and HEASARCs software package FTOOLS ${ }^{2}$ (Blackburn 1995). Spectral fitting was performed in XSPEC (Arnaud 1996) v12.7.0, using the Tübingen-Boulder ISM absorption model (TBabs in XSPEC), together with the photoelectric absorption cross-sections from Balucinska-Church \& McCammon (1992) and ISM abundances from Wilms et al. (2000). Additionally, data from the XMM-Newton optical monitor (OM; Mason et al. 2001) was processed using the standard omichain pipeline.

\section{Results}

A new X-ray source was discovered serendipitously in the $\mathrm{ToO}$ observation in August 2011, which was triggered to constrain the SSS turn-off of a different M 31 disk nova (see Henze et al., in prep.). This new source was found at RA $=00 \mathrm{~h} 44 \mathrm{~m} 01.83 \mathrm{~s}$,

\footnotetext{
${ }^{1} \mathrm{http}: / / \mathrm{xmm}$. esac.esa.int/external/xmm_data_analysis/

2 http://heasarc.gsfc.nasa.gov/ftools/
}

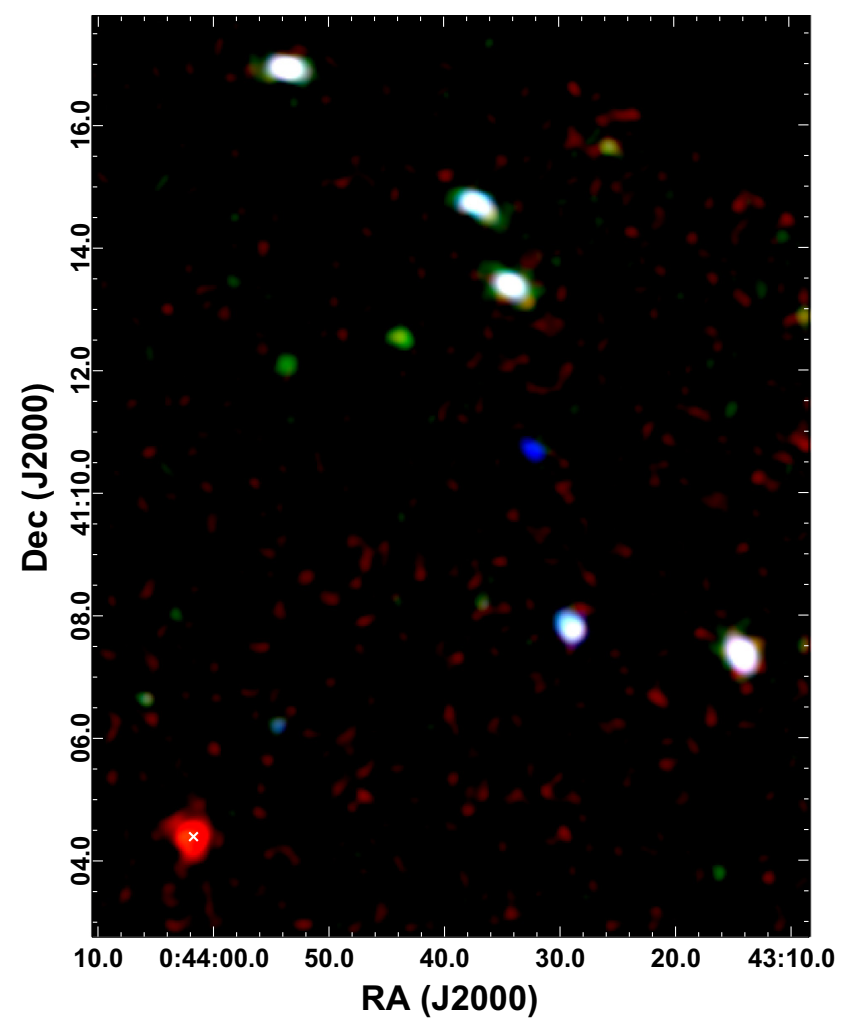

Fig. 1. XMM-Newton EPIC RGB cut-out of observation 0655620401 showing M31N 2008-05d in relation to a characteristic X-ray constellation south-east of the M 31 centre. Energy bands are $(0.2-0.5) \mathrm{keV}$, $(0.5-1.0) \mathrm{keV}$, and (1.0-2.0) keV for red, green, and blue channels, respectively. M31N 2008-05d is visible as a red source in the lower left corner of the field. The small white cross gives the position of the optical nova. The data in each colour band were binned in $2^{\prime \prime} \times 2^{\prime \prime}$ pixels and smoothed using a Gaussian of FWHM 10".

Dec $=+41^{\circ} 04^{\prime} 23^{\prime \prime} \cdot 27(\mathrm{~J} 2000)$, which is within $1^{\prime \prime} \cdot 3$ of the position of the CN M31N 2008-05d (see Fig. 1) as given in the discovery alert by Ovcharov et al. (2008). Within their uncertainties (optical: 0'3, X-ray: 1'.9), both positions are in agreement. Therefore, we identified the new source as the X-ray counterpart of the CN. No X-ray source had been known at this position from previous X-ray surveys of M 31 (see Stiele et al. 2011, and references therein).

We checked archival X-ray data and found that the source was not detected during a $22 \mathrm{ks}$ XMM-Newton ToO observation on 2008-07-18.26 UT (see also Pietsch et al. 2008), 51 days after the optical discovery. The corresponding upper-limit luminosity, given in Table 1, was determined from the more sensitive EPIC pn detector. The position of the source was included 


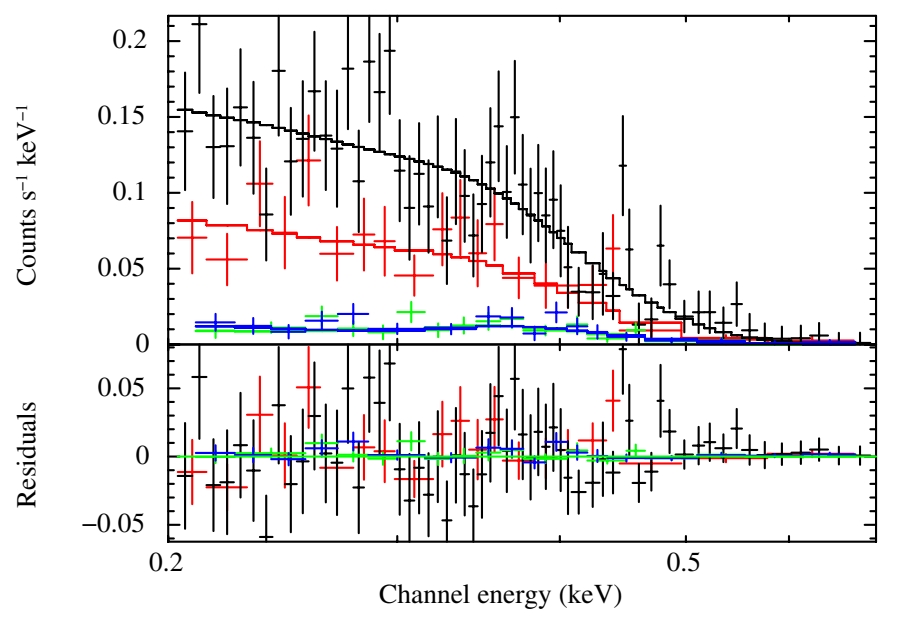

Fig. 2. XMM-Newton EPIC spectra of M31N 2008-05d for ObsIDs 0655620301 (pn: red) and 0655620401 (pn: black, MOS 1: green, MOS 2: blue). Blackbody fits are shown as solid lines in corresponding colours, with the lower panel giving the fit residuals.

in the field of view of further five X-ray observations with Chandra and Swift between this upper limit and our discovery: Chandra ACIS-I ObsIDs 11256 (20 ks; 2009-09-17.38 UT) and 11252 (59 ks; 2009-09-19.41); Swift ObsIDs 00037726002 (4.8 ks; 2008-07-22.07), 0003772503 (440 s; 2009-08-23.30), and 00037725004 (2.6 ks; 2009-09-04.56). However, the upper limits from these observations, assuming a $30 \mathrm{eV}$ blackbody spectrum (see below), are at least one order of magnitude brighter than the discovery luminosity. This is caused by the short exposure times and relatively small collecting area in the case of Swift and the comparatively poor spectral response below $1 \mathrm{keV}$ of the Chandra ACIS-I detector, when both are compared to XMM-Newton.

The second ToO observation was triggered in January 2012, to follow up the nova counterpart which was found to still be active. The XMM-Newton EPIC spectra of both detections are shown in Fig. 2 and compatible within their uncertainties. The merged spectrum could be described using an absorbed blackbody model with best-fit parameters $k T=32 \pm 6 \mathrm{eV}$ and $N_{\mathrm{H}}=$ $\left(1.2_{-0.4}^{+0.5}\right) \times 10^{21} \mathrm{~cm}^{-2}$, allowing us to classify the source as a SSS. The SSS nature of the source provides further evidence to identify it with the optical nova. Unabsorbed luminosities inferred from the spectral model are given in Table 1, together with "equivalent luminosities" $\left(L_{50}\right)$ which assume a generic $50 \mathrm{eV}$ blackbody spectrum with Galactic foreground absorption $\left(N_{\mathrm{H}} \sim 6.7 \times 10^{20} \mathrm{~cm}^{-2}\right)$ and are used, as in HPH2011, to compare luminosities to earlier works (e.g. Pietsch et al. 2007; Henze et al. 2010). The blackbody parametrisation is discussed in Sect. 4.

No counterpart was detected in the XMM-Newton OM data, with upper limits of $\sim 21.5$ and $\sim 20.0 \mathrm{AB}$ magnitudes in the uvw1 and uvw2 bands, respectively, for both ToO observations.

Owing to the long gap between the only upper limit and the first detection, the SSS turn-on time of the nova is only marginally constrained to $t_{\mathrm{On}}=(606 \pm 555) \mathrm{d}$. The turn-off of the SSS state is yet to happen $1334 \mathrm{~d}$ after the optical detection.

The X-ray short-term light curve of nova M31N 2008-05d displayed variability during both ToO pointings (Fig. 3). The first observation (ObsID 0655620301) showed a relatively stable count rate during the initial $\sim 10 \mathrm{ks}$, after which there are indications for a dip that lasted $\sim 4 \mathrm{ks}$. After this possible dip, during which the count rate dropped by a factor of $\sim 2$, the count rate
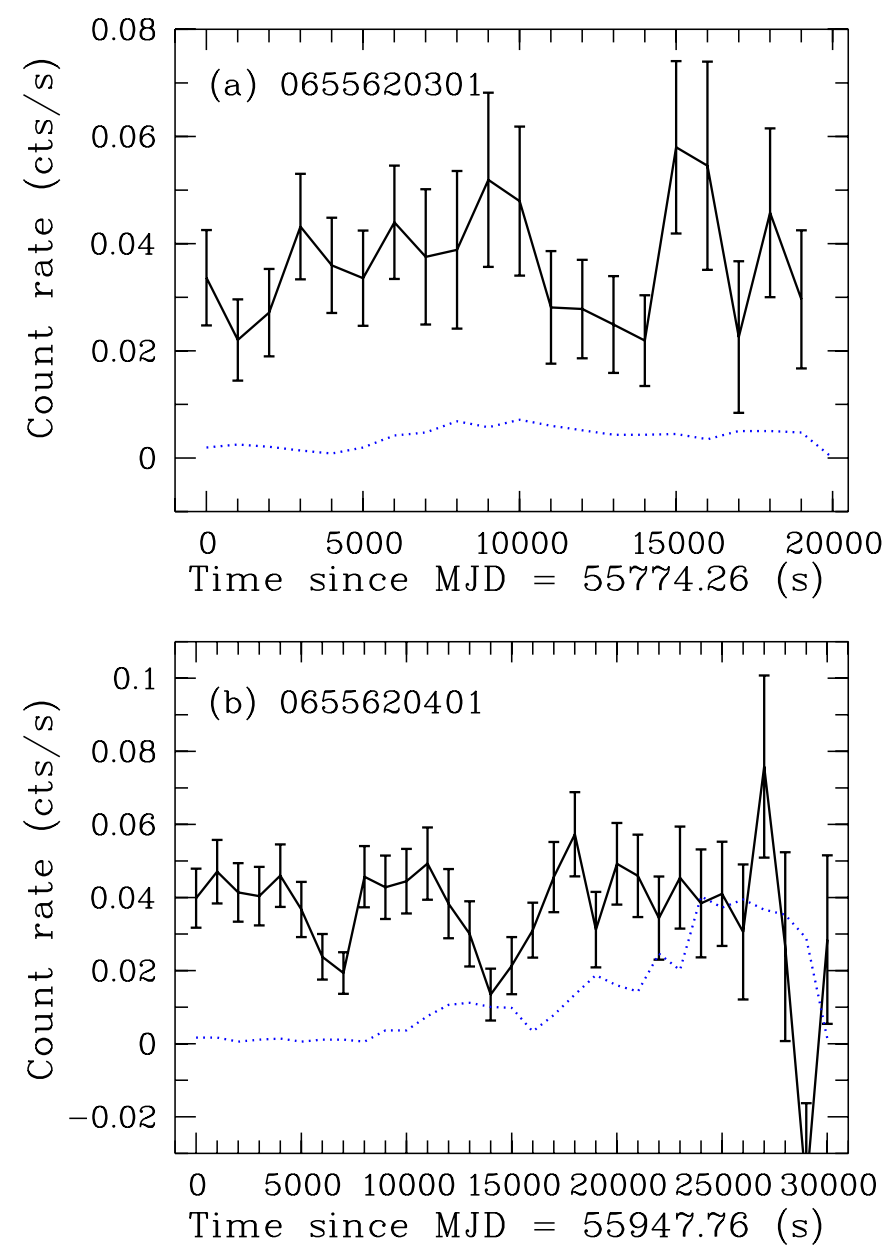

Fig. 3. Short-term, background-subtracted EPIC pn X-ray light curves of nova M31N 2008-05d in the $0.2-1.0 \mathrm{keV}$ range during XMM-Newton observations 0655620301 (upper panel, a)) and 0655620401 (lower panel, b)) with $1000 \mathrm{~s}$ binning and $3 \sigma$ error bars. In blue, the areacorrected, background light curves in the same energy range are shown for a source-free region close to the nova.

seemed to return to the previous level. Although the ingress of the dip shows a certain resemblance to the soft-background light curve, its general shape cannot be explained by the background alone (see Fig. 3a).

In the second observation, the dip features are considerably more significant. Two pronounced dips can easily be identified in the first $20 \mathrm{ks}$ of the light curve, as shown in Fig. 3b. While the first dip, by a factor of $\sim 2$, lasted for $\sim 3 \mathrm{ks}$ and appeared asymmetric, the second, possibly deeper dip had a more symmetric profile and was $\sim 5 \mathrm{ks}$ long.

The first dip happened in its entirety during the initial $\sim 8 \mathrm{ks}$ of the observation, which were characterised by low background (see the blue background light curve in Fig. 3b). In contrast, the second dip took place during a time of higher background, but shows neither suspicious symmetry nor anti-symmetry with the background light curve. Measurements during the last $\sim 7 \mathrm{ks}$ of the observation are unreliable owing to the presence of a very high background and are only shown for completeness.

We found that the possible dip during the first observation is only mildly significant within $(1-2) \sigma$ with respect to the rest of the light curve. In constrast, both dips during the second ToO pointing exceed a $3 \sigma$ significance level in two bins each. This difference might be connected to the otherwise stable brightness level of M31N 2008-05d during the second observation, 
but it ultimately means that the evidence of a dipping SSS light curve is solely based on this second observation, with the first light curve only providing a hint about this similar previous behaviour. The identified dip features can be attributed to the nova itself and are unlikely to stem from background variations. No periodicities were found in either light curve within the range of $10^{-4}-10^{-1} \mathrm{~Hz}$ using the HEASoft powspec tool.

\section{Discussion}

M31N 2008-05d was discovered in the south-eastern part of the M31 disk. Although its position lies broadly along the minor axis of the galaxy, the nova is sufficiently far away from the bulge for projection effects to have no effect on its classification as a disk nova. The object does not appear to be associated with a bright spiral arm of M 31 .

The relationships between the X-ray parameters of M31N 2008-05d follow the trends found in the M31 sample of novae with SSS counterparts by HPH2011. The long SSS phase together with the low blackbody temperature point towards a slowly evolving nova on a low-mass WD (see Hachisu \& Kato 2010, HPH2011). This interpretation also fits the slow evolution in the optical reported by Henze et al. (2008a). Although M31 disk novae are suspected to be on average hotter SSSs (mean blackbody $k T=56 \mathrm{eV}$ in HPH2011) than bulge novae, the temperature derived for M31N 2008-05d lies within the lowenergy tail of the current, relatively broad temperature distribution (see Fig. 13 in HPH2011).

Unfortunately, the OM non-detection does not provide additional constraints on the underlying binary. In the optical blue/ultraviolet regime, the emission of a $\mathrm{CN}$ system in quiescence is dominated by the accretion disk rather than by either the main-sequence or red-giant secondary star (e.g. Darnley et al. 2012). While M31N 2008-05d is not yet in quiescence, the emission from the WD photosphere has been effectively shifted out of the optical/ultraviolet range (see Hachisu \& Kato 2006), thereby creating the SSS. Owing to the distance of M 31, our OM upper limits do not provide sufficient sensitivity to distinguish between the CN secondary star classes (compare also Bode et al. 2009).

Novae with dipping short-term X-ray light curves are rare in M31. The only comparable object was nova M31N 2006-04a, whose SSS light curve displayed three deep minima during a 20 ks XMM-Newton observation (Henze et al. 2010). However, Henze et al. (2010) reported for M31N 2006-04a an apparent periodicity, which appeared to indicate the binary period of the system. In contrast, the aperiodic nature and different shape of the dips observed in M31N 2008-05d make an association with the WD rotation or the orbital period of the binary system unlikely. To investigate the nature of the dips further, we divided the light curve of observation 0655620401 (see Fig. 3b) into two energy bands based on the SSS spectrum: $0.2-0.35 \mathrm{keV}$ and $0.35-0.6 \mathrm{keV}$. Above $0.6 \mathrm{keV}$, we detected no photons from the source. The resulting light curves for the relatively soft and hard ranges are shown in Fig. 4.

The figure illustrates that the dips are almost exclusively confined to energies below $0.35 \mathrm{keV}$. This is a strong hint that the dips might be caused by photoelectric absorption in intervening material, which has a smaller effect at higher energies. In this scenario, the material, possibly gas clouds, would be crossing the line of sight randomly. The different length and shape of the individual dips suggests that the absorbers have wide ranges of sizes and/or velocities. We also extracted and fitted two separate spectra for the dipping and non-dipping times during observation 0655620401. However, although we found that the

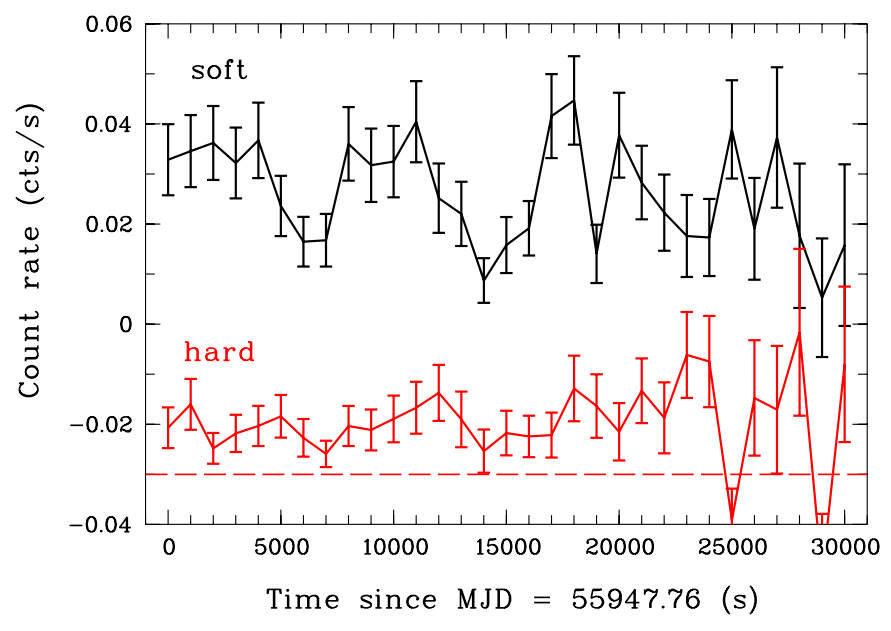

Fig. 4. Same as Fig. $3 b$ for energy bands $0.2-0.35 \mathrm{keV}$ (soft, black) and $0.35-0.6 \mathrm{keV}$ (hard, red). The lower light curve includes an offset zero level (dashed red line) for better readability.

best-fit $N_{\mathrm{H}}$ is larger during the dips by about a factor of two ( 1.9 vs $1.0 \times 10^{21} \mathrm{~cm}^{-2}$ ), owing to the low count rates the errors are large and both extinction values are still compatible on the $1 \sigma$ level.

However, high-resolution spectra of Galactic novae (e.g. Nelson et al. 2008; Ness et al. 2011, 2012) emphasize that blackbody fits only provide a qualitative parametrisation of SSS spectra and not a physically realistic model (see also Greiner et al. 1991; Kahabka \& van den Heuvel 1997). Therefore, the variability of M31N 2008-05d could also originate in the changing properties of individual emission or absorption features that are not considered by our simple model. One example for such an effect is the explanation of variability caused by a variation in the optical depth of the O I absorption edge in the SSS state of the Galactic nova RS Ophiuchi (Ness et al. 2007).

While dipping SSS light curves are rare in M31 novae, Galactic novae have been observed to show dip-like or eclipse features in X-rays on relatively short timescales. Most of these objects behave however, in a significantly different way from M31N 2008-05d (see e.g. Ness et al. 2003; Sala et al. 2008; Ness et al. 2011). The only exception is the recurrent nova U Sco, for which similar dips to those in Fig. 3b were found in its 2010 outburst by Ness et al. (2012). These authors note that the X-ray dips, shown in their Fig. 2, could originate from absorption by "clumpy absorbing material that intersects the line of sight while moving along highly elliptical trajectories". Interestingly, Ness et al. (2012) report the dips to be present only in an early state of the SSS phase (22.9 d after outburst) and to have already disappeared in a second observation on day 34.9 , a behaviour that they interpret as a signature of "significant progress in the formation of the (accretion) disk".

If a similar picture applied to M31N 2008-05d, the timescales involved would be considerably longer, since the M 31 nova experienced its dips at a much later stage: more than three years after outburst. However, M31N 2008-05d shows an overall slower evolution in X-rays and optical than U Sco. Little is known to date about how quickly $\mathrm{CN}$ accretion disks reform (e.g. Retter et al. 1998; Hernanz \& Sala 2002; Ness et al. 2012). M31N 2008-05d might in respect of disk reformation resemble $\mathrm{U}$ Sco on longer timescales. In this case, the high-inclination nature of the U Sco binary system $\left(83^{\circ} \pm 3^{\circ}\right.$, Thoroughgood et al. 2001) suggests that we might also be viewing M31N 2008-05d at high inclination. 
An alternative scenario to explain the dips could be absorption in the ejected nova shell. Inhomogeneous and clumpy ejecta have been observed for several Galactic novae in their resolved nebular remnants (e.g. Gill \& O'Brien 2000), optical spectra (e.g. Williams et al. 1991), and even in X-rays (e.g. Balman \& Ögelman 1999). Evidence of density inhomogeneities in the ejected material was also discussed for U Sco based on optical data (Diaz et al. 2010). Ness et al. (2012) ruled out the possibility that the dips they found in U Sco were caused by the inhomogeneous ejecta, on the basis that these clumps are expected to be highly ionized and therefore should not obscure soft X-rays via photoelectric absorption. With regard to the later evolutionary stage of M31N 2008-05d, this argument might not, however hold in our case. Furthermore, the possibility of Thomson scattering within the hot ejecta was also rejected by Ness et al. (2012) for U Sco, as it would have led to optical/UV dips detectable in their simultaneous XMM-Newton OM fast-mode light curves. For M31N 2008-05d, owing to the OM non-detection and novae in M 31 being more distant, this cross check is unfortunately impossible. However, the energy dependence shown in Fig. 4 is strongly indicative of photoelectric absorption.

Another possible scenario might be the occultation of the WD by cold, clumpy material caused by the impingement of a high-accretion rate stream on the accretion disk, as discussed by Schandl et al. (1997) for the canonical SSS CAL 87. However, applying this model as constructed for CAL 87 to M31N 2008-05d would result in a permanent attenuation of the WD by a kind of curtain of spray material. While this curtain would extend beyond the accretion disk towards a large vertical height, and thereby permit a larger range of inclination angles, the permanence of the occultation differs decidedly from the distinct dips we observed for our object. Nevertheless, it is possible that an inhomogeneously extended accretion stream or streamimpact spray could cause the observed non-periodic X-ray variability. Therefore, the model of Schandl et al. (1997) might help to explain the light curve of M31N 2008-05d within a more sophisticated framework, the construction of which is beyond the scope of this paper.

The high nova rate and the proximity of M31 have not only allowed us to construct an unparalleled statistical sample but also provided us with a number of unusual objects. Both features enable our understanding to advance by revealing patterns and exceptions within the nova population. A continuing X-ray monitoring of M 31 is therefore strongly needed to provide a sufficiently large database for future discoveries.

Acknowledgements. We thank the anonymous referee for her/his constructive comments and suggestions. The X-ray work is based in part on observations with $X M M-N e w t o n$, an ESA Science Mission with instruments and contributions directly funded by ESA Member States and NASA. The XMM-Newton project is supported by the Bundesministerium für Wirtschaft und Technologie / Deutsches Zentrum für Luft- und Raumfahrt (BMWI/DLR FKZ 50 OX 0001) and the Max-Planck Society. M. Henze acknowledges support from the BMWI/DLR, FKZ 50 OR 1010. GS acknowledges the MICINN grants AYA2010-15685 and AYA2011-23102, and the ESF EUROCORES Program EuroGENESIS through the MICINN grant EUI2009-04167. M. Hernanz acknowledges support from the MICINN grant AYA2011-24704.

\section{References}

Arnaud, K. A. 1996, in Astronomical Data Analysis Software and Systems V,

eds. G. H. Jacoby, \& J. Barnes, ASP Conf. Ser., 101, 17

Balman, Ş., \& Ögelman, H. B. 1999, ApJ, 518, L111

Balucinska-Church, M., \& McCammon, D. 1992, ApJ, 400, 699

Blackburn, J. K. 1995, in Astronomical Data Analysis Software and Systems IV, eds. R. A. Shaw, H. E. Payne, \& J. J. E. Hayes, ASP Conf. Ser., 77, 367

Bode, M. F., \& Evans, A. 2008, Classical Novae, eds. M. F. Bode, \& A. Evans Bode, M. F., Darnley, M. J., Shafter, A. W., et al. 2009, ApJ, 705, 1056

Darnley, M. J., Ribeiro, V. A. R. M., Bode, M. F., Hounsell, R. A., \& Williams, R. P. 2012, ApJ, 746, 61

Della Valle, M., \& Livio, M. 1998, ApJ, 506, 818

Della Valle, M., Bianchini, A., Livio, M., \& Orio, M. 1992, A\&A, 266, 232

Diaz, M. P., Williams, R. E., Luna, G. J., Moraes, M., \& Takeda, L. 2010, AJ 140,1860

Duerbeck, H. W. 1990, in Physics of Classical Novae, eds. A. Cassatella, \& R. Viotti (Berlin: Springer Verlag), IAU Colloq., 122, Lect. Notes Phys., 369, 34

Gabriel, C., Denby, M., Fyfe, D. J., et al. 2004, in Astronomical Data Analysis Software and Systems (ADASS) XIII, eds. F. Ochsenbein, M. G. Allen, \& D. Egret, ASP Conf. Ser., 314, 759

Gill, C. D., \& O'Brien, T. J. 2000, MNRAS, 314, 175

Greiner, J., Hasinger, G., \& Kahabka, P. 1991, A\&A, 246, L17

Hachisu, I., \& Kato, M. 2006, ApJS, 167, 59

Hachisu, I., \& Kato, M. 2010, ApJ, 709, 680

Henze, M., Burwitz, V., Pietsch, W., et al. 2008a, The Astronomer's Telegram, 1567,1

Henze, M., Pietsch, W., Burwitz, V., et al. 2008b, The Astronomer's Telegram, 1602,1

Henze, M., Pietsch, W., Haberl, F., et al. 2010, A\&A, 523, A89

Henze, M., Pietsch, W., Haberl, F., et al. 2011, A\&A, 533, A52 (HPH2011)

Hernanz, M., \& Sala, G. 2002, Science, 298, 393

Holland, S. 1998, AJ, 115, 1916

Jansen, F., Lumb, D., Altieri, B., et al. 2001, A\&A, 365, L1

Kahabka, P., \& van den Heuvel, E. P. J. 1997, ARA\&A, 35, 69

Krautter, J. 2008, in ASP Conf. Ser. 401, eds. A. Evans, M. F. Bode, T. J. O’Brien, \& M. J. Darnley, 139

Mason, K. O., Breeveld, A., Much, R., et al. 2001, A\&A, 365, L36

Nelson, T., Orio, M., Cassinelli, J. P., et al. 2008, ApJ, 673, 1067

Ness, J., Starrfield, S., Burwitz, V., et al. 2003, ApJ, 594, L127

Ness, J.-U., Starrfield, S., Beardmore, A. P., et al. 2007, ApJ, 665, 1334

Ness, J.-U., Osborne, J. P., Dobrotka, A., et al. 2011, ApJ, 733, 70

Ness, J.-U., Schaefer, B. E., Dobrotka, A., et al. 2012, ApJ, 745, 43

Ovcharov, E., Valcheva, A., Latev, G., et al. 2008, The Astronomer's Telegram, 1563, 1

Pietsch, W., Fliri, J., Freyberg, M. J., et al. 2005, A\&A, 442, 879

Pietsch, W., Haberl, F., Sala, G., et al. 2007, A\&A, 465, 375

Pietsch, W., Freyberg, M., Haberl, F., Henze, M., \& Stiele, H. 2008, The Astronomer's Telegram, 1647, 1

Retter, A., Leibowitz, E. M., \& Kovo-Kariti, O. 1998, MNRAS, 293, 145

Sala, G., \& Hernanz, M. 2005, A\&A, 439, 1061

Sala, G., Hernanz, M., Ferri, C., \& Greiner, J. 2008, ApJ, 675, L93

Schandl, S., Meyer-Hofmeister, E., \& Meyer, F. 1997, A\&A, 318, 73

Schwarz, G. J., Ness, J.-U., Osborne, J. P., et al. 2011, ApJS, 197, 31

Stanek, K. Z., \& Garnavich, P. M. 1998, ApJ, 503, L131

Stark, A. A., Gammie, C. F., Wilson, R. W., et al. 1992, ApJS, 79, 77

Starrfield, S. 1989, in Classical Novae, 39

Starrfield, S., Sparks, W. M., \& Truran, J. W. 1974, ApJS, 28, 247

Stiele, H., Pietsch, W., Haberl, F., et al. 2011, A\&A, 534, A55

Strüder, L., Briel, U., Dennerl, K., et al. 2001, A\&A, 365, L18

Thoroughgood, T. D., Dhillon, V. S., Littlefair, S. P., Marsh, T. R., \& Smith, D. A. 2001, MNRAS, 327, 1323

Turner, M. J. L., Abbey, A., Arnaud, M., et al. 2001, A\&A, 365, L27

Williams, R. E., Hamuy, M., Phillips, M. M., et al. 1991, ApJ, 376, 721

Wilms, J., Allen, A., \& McCray, R. 2000, ApJ, 542, 914 